\title{
Case report of necrotizing sialometaplasia
}

\author{
Antonio Bascones-Martínez ${ }^{1}$, Marta Muñoz-Corcuera ${ }^{2}$, Rocío Cerero-Lapiedra ${ }^{3}$, Jaime Bascones-Ilun- \\ dáin ${ }^{4}$, Germán Esparza-Gómez ${ }^{3}$
}

\author{
${ }^{1}$ Chairman of Oral Medicine and Periodontics. Medicine and Orofacial Surgery Department, School of Dentistry, Complutense \\ University of Madrid, Spain \\ ${ }^{2}$ Dentistry Graduate. PhD Student. Fellow on Education Department FPU programme, Medicine and Orofacial Surgery Depart- \\ ment, School of Dentistry, Complutense University of Madrid, Spain \\ ${ }^{3}$ Professor of Oral Medicine. Medicine and Orofacial Surgery Department, School of Dentistry, Complutense University of \\ Madrid, Spain \\ ${ }^{4}$ Professor of Endodontics. Conservative Department, School of Dentistry, Complutense University of Madrid Spain
}

Correspondence:

Departamento de Medicina y Cirugía Bucofacial

Facultad de Odontología

Universidad Complutense de Madrid. Spain

Plaza Ramón y Cajal, s/n

28040 Madrid. Spain

antbasco@odon.ucm.es

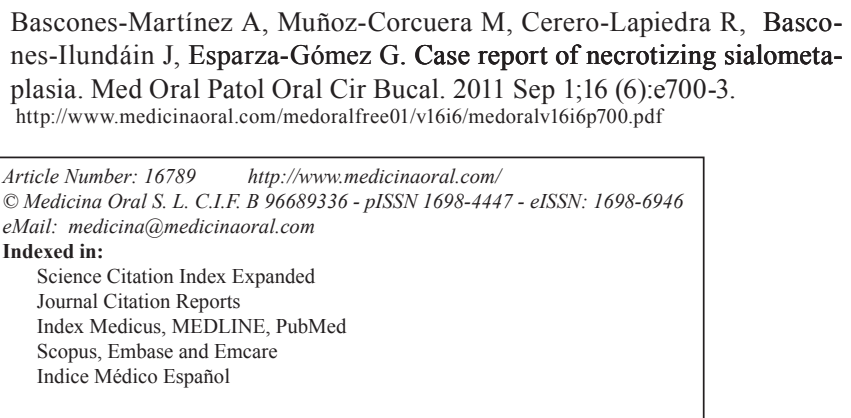

\begin{abstract}
Introduction: Necrotizing sialometaplasia is a rare inflammatory necrotizing reactive process that can involve minor and major salivary glands. Gland tissue ischaemia has been proposed as the aetiology.

Case Report: A 21-year-old woman was referred with a 1-week history of bilateral painful swelling of the palate, with the development of two deep ulcers after the first 3 days. Ulcer edges were elevated but not indurated, and the bases had a necrotic appearance. An incisional biopsy of the margin of one ulcer confirmed the diagnosis of necrotizing sialometaplasia. The ulcers spontaneously healed within 8 weeks without treatment.

Discussion: The clinical and histological similarity between this entity and a malignant lesion implies a risk of unnecessary or inadequate treatment. This case illustrates the need for an incisional biopsy to be analyzed by an experienced pathologist to establish a correct diagnosis.
\end{abstract}

Key words: Necrotizing sialometaplasia, oral ulcers, minor salivary glands, palate, biopsy. 


\section{Introduction}

Necrotizing sialometaplasia (NS) was first described in 1973 by Abrams et al. as an inflammatory necrotizing reactive process affecting minor salivary glands of the hard palate (1). They proposed the following histopathological criteria: necrosis of acinary cells of seromucinous glands; squamous metaplasia of salivary ductal epithelial and acini; pseudoepitheliomatous hyperplasia of the epithelium lining the gland; mucous release; inflammatory response associated with granulation tissue in or around the glands; intact lobular architecture; and histologically benign nuclear morphology, although normal mitoses can sometimes be observed (1).

There have since been numerous case reports of this lesion (2-11), not only on the hard palate but at all sites with salivary tissue, including upper and lower lip $(2,4,7)$, maxillary sinus $(2,4)$, floor of mouth (2), tongue $(2,4)$, retromolar area (4), oral mucosa (4), tonsillar fossa (4), major salivary glands (4), nasal cavity (4), incisor canal (4), larynx (4), soft palate $(4-6,8,11)$ and soft-hard palate junction $(4,5)$.

The histological characteristics proposed by Abrams et al. were modified by Brannon (1991) (4), who described a predominance of coagulative necrosis of acini in early lesions and of squamous metaplasia and reactive fibrosis in later lesions. Anneroth, Hansen and Imbery, Edwards (6) described five histological stages of NS: infarction (necrosis), sequestration, ulceration, repair and healing. NS is an uncommon, benign and self-limiting reactive inflammatory process involving minor and major salivary glands. The most frequent NS site is the hard palate $(58 \%)$, mainly manifesting as a deep crateriform ulcer with indurated and well-defined edges or, less often, as a non-ulcerated mass $(2,4)$, generally unilateral but bilateral in around $12 \%$ of cases (3), as in the present patient. Bone involvement has been reported in only a few cases (4). The lesion can be painful or asymptomatic, while some patients present with fever, malaise or paraesthesia/anaesthesia of the area (4). The lesion is most frequently detected in Caucasian males aged between 40 and 50 years (4).

The aetiology of NS has not been fully elucidated, but an underlying cause appears to be gland tissue ischemia (4), leading to infarction and subsequent necrosis of the tissue, followed by repair and metaplasia (8). Numerous risk factors have been described, including: local trauma, e.g., surgery, intubation, poor-fitting prostheses, fellatio or local pressure $(2,4,6,8)$; alcohol $(4,5)$, tobacco $(4,5,9)$ or cocaine $(6)$ use; radiation $(6)$; respiratory infections or allergies (4); previous adenoidectomy or surgery for other lesions (e.g., tumours, mucoceles) $(5,7,11)$; adjacent tumours or other lesions producing compression and ischemia (4); bulimia and chronic vomiting $(8,10)$; diabetes $(8)$; and drepanocytic anaemia, with an increase in blood viscosity that favours ischemia (3). All of these factors can compromise the supply of blood to affected glands.

Application of local dental anaesthesia to the hard palate is thought to play a role in this disease due to needle-induced trauma and vasoconstrictive effects $(2,4,6)$. In 1996, Shigematsu et al. (12) found a relationship between the repeated application of local anaesthesia into rat palate and histological changes similar to those observed in NS. In the present patient, the only predisposing factor was tobacco use.

Management of these lesions should always include an incisional biopsy and close follow-up until their complete disappearance, administering analgesics in cases of pain. There are usually no recurrences or anatomic or functional sequelae $(6,11)$. NS lesions heal by second intention without treatment within 4-10 weeks $(4,6,11)$, and the healing time is primarily related to the size rather than the nature of the lesions (6).

The importance of this clinical entity lies in its clinical and histological similarity to a malignant lesion. Because of the severity of the ulceration and tissue destruction over a short time period, it is frequently misdiagnosed as mucoepidermoid carcinoma, squamous cell carcinoma or cystic adenoid carcinoma, leading to unnecessary treatments ranging from conservative excision to total maxillectomy $(4,5,8,10)$. Analysis by an experienced pathologist of an incisional biopsy is therefore essential to establish a correct diagnosis (9).

\section{Case Report}

\section{Clinical and pathological characteristics}

A 21-year-old woman, smoker of 10 cigarettes/day but with no other predisposing factors, was referred to our Department by her general dental practitioner with a one-week history of bilateral palatal swellings that had developed into two deep ulcers after the first three days. The swellings had been painful and her temperature had risen to $38^{\circ} \mathrm{C}$ during the initial three-day period, but both the pain and fever disappeared with the appearance of the ulcers.

Clinical examination revealed two deep ulcers $(3 \times 1 \mathrm{~cm}$ and $2 \times 1 \mathrm{~cm}$ ), one on either side of the midline of the hard palate (Fig. 1). The edges of the lesions were elevated but not indurated, and the base was covered with necrotic debris. No pain was caused by the examination, and no abnormities were observed in the rest of the oral cavity. Haematological and radiographic findings were normal.

An incisional biopsy of the peripheral margin of one of the ulcers was taken under local anaesthesia. Microscopic study of histological sections revealed mucosa composed of parakeratotic, stratified squamous epithelium. The submucosa contained numerous degenerating mucus-producing salivary glands with mucus spillage. Some areas showed extensive necrosis of glandular aci- 


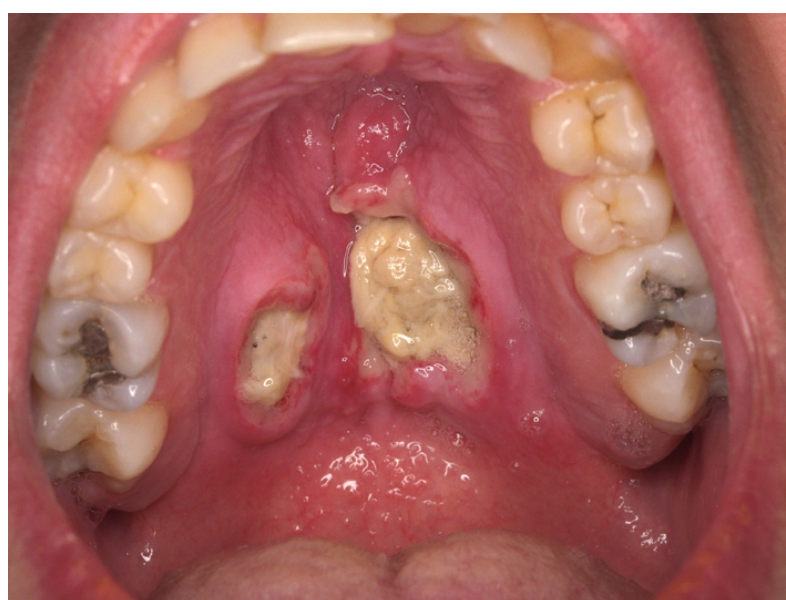

Fig. 1. Palatal ulcers at presentation.

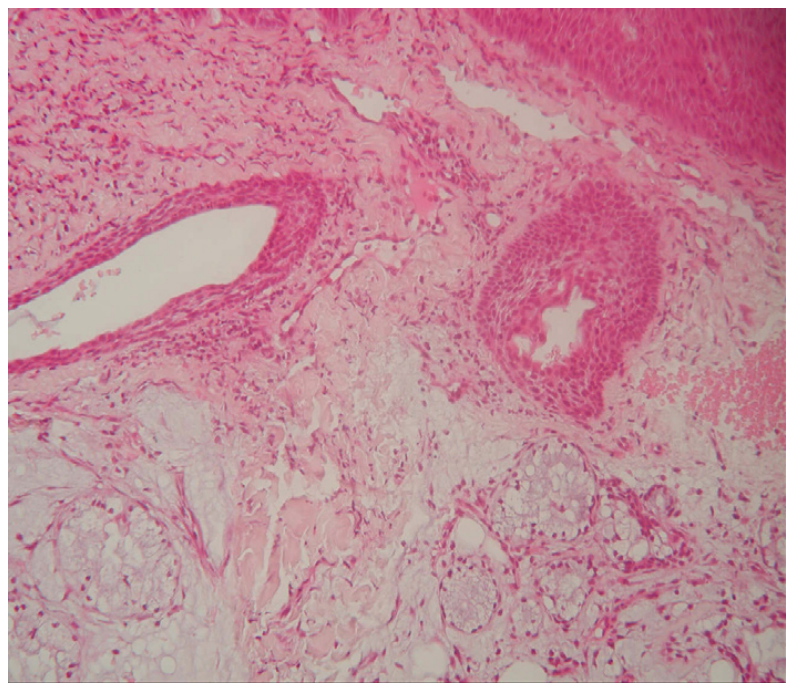

Fig. 2. Necrosis of acini and acute inflammatory infiltrate (HE 20x).

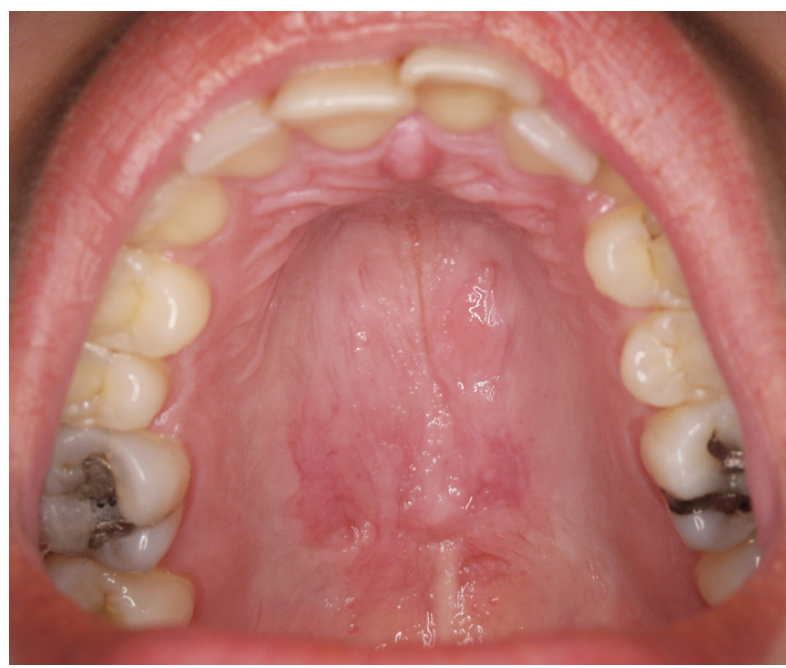

Fig. 3. Lesions resolved at 8 weeks from the onset. ni and squamous ductal metaplasia (Fig. 2). The connective tissue was infiltrated by a mixed inflammatory infiltrate. All of these histopathological findings were consistent with the diagnosis of necrotizing sialometaplasia.

The ulcers resolved spontaneously without treatment over the following 8 weeks (Fig. 3).

\section{Discussion}

The differential diagnosis of necrotizing sialometaplasia should consider other ulcerous and erosive lesions, including those of traumatic or inflammatory/infectious aetiology, e.g., dental fissures, major aphthae, tuberculosis, tertiary syphilis or deep fungal infection in patients with AIDS or under immunosuppressive treatment $(6,8,11)$, and those of cancerous origin, e.g., squamous cell carcinoma, mucoepidermoid carcinoma, adenoid carcinoma, leukaemia, non-Hodgkin lymphoma or sarcoma $(6,8,13,14)$.

NS is differentiated from tuberculosis, syphilis and fungal ulcers by means of serologic tests and by staining histopathological samples for fungi, bacilli and bacteria (6). Histological criteria to distinguish NS from a malignancy are: (1) preserved general lobular morphology, (2) bland appearance of squamous islands or nests with no cytological evidence of malignancy and (3) no findings of residual ductal lumina in any nest $(4,14)$. Reactive atypias are occasionally observed in nests or islets, and the diagnosis must then be confirmed by the general lobular appearance and the presence of intraepithelial inflammation in squamous nests (4). When samples are too small to permit study of the general architecture, this entity can be confused with a squamous cell or mucoepidermoid carcinoma $(10,14)$. In addition, the metaplastic and hyperplastic changes can be florid, and the reparative fibrosis can be confused with infiltrate (10). Account should be taken of the aforementioned predominance of coagulative necrosis of acini in early lesions and of squamous metaplasia and reactive fibrosis in later lesions (4).

Rizkalla and Toner (14) studied the usefulness of myoepithelial markers and cytokeratin subtype expression to distinguish NS from squamous cell and mucoepidermoid carcinomas by immunohistochemistry. They concluded that expression of calponin and smooth muscle actin in myoepithelial cells from the periphery of squamous islets and focal staining with cytokeratin 7 are characteristic of NS but not normally expressed in carcinomas. This diagnostic tool may be especially useful when the sample is of inadequate size.

The possibility of a subacute necrotizing sialadenitis should also be taken into account, although some authors consider it to lie within the spectrum of NS $(8,15)$. According to Fowler et al. (15), it is a non-specific acute inflammatory condition of unknown origin, histologi- 
cally characterized by focal acinar necrosis (secondary to the inflammation) and atrophy of duct cells, without ductal metaplasia, pseudoepitheliomatous hyperplasia or fibrosis $(8,15)$. It manifests as non-ulcerated erythematous nodular lesions on the palate accompanied by acute pain and has been reported in young people living in groups $(8,15)$. The lesions usually last for around two weeks and it has been suggested that the aetiology may be infectious (viral) or allergic (15).

\section{References}

References with links to Crossref - DOI

1. Abrams AM, Melrose RJ, Howell FV. Necrotizing sialometaplasia. A disease simulating malignancy. Cancer. 1973;32:130-5.

2. Van der Wal JE, Van der Waal I. Necrotizing sialometaplasia: report of 12 new cases. Br J Oral Maxillofac Surg. 1990;28:326-8.

3. Mandel L, Kaynar A, DeChiara S. Necrotizing sialometaplasia in a patient with sickle-cell anemia. J Oral Maxillofac Surg. 1991;49:757-9.

4. Brannon RB, Fowler CB, Hartman KS. Necrotizing sialometaplasia. A clinicopathologic study of sixty-nine cases and review of the literature. Oral Surg Oral Med Oral Pathol. 1991;72:317-25.

5. Abaza NA, Abaza MM, Sedhom AW. Necrotizing sialometaplasia: a review and two case reports. Compendium. 1992;13:12,14,16-8.

6. Imbery TA, Edwards PA. Necrotizing sialometaplasia: literature review and cas reports. J Am Dent Assoc. 1996;127:1087-92.

7. Pulse CL, Lebovics RS, Zegarelli DJ. Necrotizing sialometaplasia: report of a case after lower lip mucocele excision. J Oral Maxillofac Surg. 2000;58:1419-21.

8. Femopase FL, Hernández SL, Gendelman H, Criscuolo MI, Lópezde-Blanc SA. Necrotizing sialometaplasia: report of five cases. Med Oral. 2004;9:304-8.

9. Komínek P, Blasch P. Necrotizing sialometaplasia: a potential diagnostic pitfall. Ear Nose Throat J. 2006;85:604-5.

10. Solomon LW, Merzianu M, Sullivan M, Rigual NR. Necrotizing sialometaplasia associated with bulimia: case report and literature review. Oral Surg Oral Med Oral Pathol Oral Radiol Endod. 2007;103:e39-42.

11. Ylikontiola L, Siponen M, Salo T, Sándor GK. Sialometaplasia of the soft palate in a 2-year-old girl. J Can Dent Assoc. 2007;73:333-6. 12. Shigematsu H, Shigematsu Y, Noguchi Y, Fujita K. Experimental study on necrotizing sialometaplasia of the palate in rats. Role of local anesthetic injections. Int J Oral Maxillofac Surg. 1996;25:23941.

13. Bascones-Martínez A, Figuero-Ruiz E, Esparza-Gómez GC. [Oral ulcers]. Med Clin (Barc). 2005;125:590-7.

14. Rizkalla H, Toner M. Necrotizing sialometaplasia versus invasive carcinoma of the head and neck: the use of myoepithelial markers and keratin subtypes as an adjunct to diagnosis. Histopathology. 2007;51:184-9.

15. Fowler CB, Brannon RB. Subacute necrotizing sialadenitis: report of 7 cases and a review of the literature. Oral Surg Oral Med Oral Pathol Oral Radiol Endod. 2000;89:600-9.

\section{Institution}

Medicine and Orofacial Surgery Department, School of Dentistry, Complutense University of Madrid, Spain 